\title{
High Expression of Galectin-1, VEGF and Increased Microvessel Density Are Associated with MELF Pattern in Stage I-III Endometrioid Endometrial Adenocarcinoma
}

\author{
Dmitry Aleksandrovich Zinovkin, Sergey Leonidovich Achinovich ${ }^{1}$, Mikhail Grigoryevich Zubritskiy ${ }^{2}$, \\ Jacqueline Linda Whatmore ${ }^{3}$, Md Zahidul Islam Pranjol ${ }^{4}$ \\ Department of Pathology, Gomel State Medical University, Gomel; 'Department of Pathology, Gomel State Clinical Oncological Dispensary, Gomel; \\ 2Department of Pathology, Grodno Regional Clinical Bureau of Pathology, Grodno, Belarus; \\ ${ }^{3}$ Institute of Biomedical and Clinical Science, University of Exeter Medical School, Exeter; \\ ${ }^{4}$ William Harvey Research Institute, Barts \& The London School of Medicine \& Dentistry Queen Mary University of London, London, UK
}

\begin{abstract}
Background: In this study, we investigate the expression of markers of angiogenesis and microvessel density (MVD) in cases of microcystic, elongated and fragmented (MELF) pattern, with its prognostic role in the survival of endometrioid endometrial adenocarcinomas (EA) patients. Methods: In this study, 100 cases of EA, 49 cases with MELF pattern and 51 without, were immunohistochemically stained for galectin-1, vascular endothelial growth factor (VEGF), and MVD. Morphometry and statistical (univariate and multivariate) analyses were performed to assess overall survival (OS) and disease-free survival. Results: The expression of VEGF $(p<.001)$ and galectin-1 $(p<.001)$, as well as MVD area $(p<.001)$ and number of vessels $/ \mathrm{mm}^{2}(p<.050)$, were significantly higher in the $+M E L F$ pattern group compared to the -MELF group. A low negative correlation between MELF-pattern and the number of days of survival $(p<.001, r=-0.47)$ was also found. A low positive correlation of MELF-pattern with galectin-1 expression $(p<.001, r=0.39)$, area of vessels $/ \mathrm{mm}^{2}(p<.001, r=0.36)$, outcome of EA $(p<.001, r=0.42)$ and VEGF expression $(p<.001, r=0.39)$ suggests potential pathological relevance of these factors in the prognosis of EA. A univariate survival analysis indicated a role for all parameters of survival. Multivariate Cox proportional hazard regression analysis revealed that only area of vessels $/ \mathrm{mm}^{2}$ (hazard ratio [HR], 1.018; 95\% confidence interval [Cl], 1.002 to 1.033), galectin-1 (HR, 1.049; 95\% Cl, 1.025 to 1.074) and VEGF (HR, 1.049; $95 \% \mathrm{Cl}, 1.022$ to 1.077 ) play key roles in OS. Conclusions: This study reports an increase in MVD, VEGF and galectin-1 expression in EA with MELF pattern and suggests that MELF pattern, along with the angiogenic profile, may be a prognostic factor in EA.
\end{abstract}

Key Words: Endometrioid endometrial carcinoma; MELF pattern; Galectin-1; Microvessel density; VEGF

Received: February 1, 2019 Revised: April 20, 2019 Accepted: May 13, 2019

Corresponding Author: Jacqueline Linda Whatmore, BSc (Hons.), PhD, Institute of Biomedical and Clinical Science, University of Exeter Medical School, Exeter, Devon EX1 2LU, UK Tel: +44-(0)1392-722944, E-mail: J.L.Whatmore@exeter.ac.uk

Corresponding Author: Md Zahidul Islam Pranjol, BSc (Hons.), MSc, PhD, William Harvey Research Institute, Barts \& The London School of Medicine \& Dentistry, Queen Mary University of London, Charterhouse Square, London EC1M 6BQ, UK

Tel: +44-(0)207-882-2272, E-mail: z.pranjol@qmul.ac.uk

Although most early-stage endometrioid endometrial adenocarcinomas (EA) have an excellent prognosis, a minority of lowgrade disease cases may have an aggressive clinical course. ${ }^{1-4} \mathrm{EAs}$ are histologically heterogeneous with myometrial invasion characterized by a microcystic, elongated and fragmented (MELF) pattern surrounded by myxoid and inflamed stroma. MELF pattern was first described by Murray et al. in 2003, but only a few studies since have reported on the biological potential of this histopathological lesion.
Most published studies have focused on the clinicopathological and immunohistochemical parameters of the cancer microenvironment in relation to the MELF pattern. However, the role of microvessels and angiogenic factors such as galectin- 1 and vascular endothelial growth factor (VEGF) in the MELF pattern has not been described. Furthermore, the role of the specific fibromyxoid stromal changes in the prognosis of survival of patients with EA remains unclear. ${ }^{6}$ The MELF pattern is known to be associated with lymphovascular invasion and lymph node metastasis, but 
no significant role of this lesion in relation to patient survival has been investigated. ${ }^{78}$ However, other studies have shown that the MELF pattern may play a negative role in the survival of patients suffering from EA. . $^{10}$

In this study, we hypothesize that there is a significant association between the MELF pattern and microvessel density (MVD), as well as angiogenic factors such as VEGF and galectin-1, which play key roles in determining poor survival of patients. Therefore, we investigated the presence of galectin-1, VEGF, and microvessels in the tumor microenvironment using an immunohistochemical technique. We also analyzed the aforementioned association and report for the first time that these criteria may be used as possible prognostic factors of EA.

\section{MATERIALS AND METHODS}

\section{Patients}

This prospective study involved women with EA who were treated in 2015 in the Grodno and Gomel regions in the Republic of Belarus. The inclusion criteria for the study were a presence of stage I-III EA (International Federation of Gynecology and Obstetrics [FIGO], 2009), hysterectomy and an absence of malignant tumors in other locations during life. EA stage IV (FIGO, 2009), Lynch syndrome, palliative treatment, a presence of synchronous and metachronous malignancies and the presence of other specific growth patterns of EA were the exclusion criteria for this study. ${ }^{11}$

The presence of the MELF pattern was confirmed independently by three pathologists (D.A.Z., S.L.A., and M.G.Z.). The pattern was identified by the presence of elongated, dilated (microcystic) and disrupted invasive tumor glands with peri-glandular fibromyxoid stromal reactions and single invasive tumor cells in the stroma around the pattern (Fig. 1).

A total of 100 out of 424 cases of EA during the study period were determined to be eligible for inclusion in this study. Patients were divided into two groups according to the presence or absence of MELF pattern. The first group included 49 subjects who had EA with stroma-specific MELF pattern (MELF positive group). The second group consisted of 51 patients who had no MELF pattern changes in the stroma (MELF negative group). The observation period was 36 months. All patients received treatment according to the National Protocols of Diagnostics and Treatment of Oncological Diseases of the Republic of Belarus. Clinicopathological characteristics of patients are presented in Table 1.

\section{Immunohistochemistry}

The primary antibodies used in this study were ready-to-use monoclonal mouse Gal-1 (Abcam, Cambridge, UK), anti-VEGF (Diagnostic Biosystems, Pleasanton, CA, USA), and anti-CD34 (Diagnostic Biosystems). An UnoVue HRP/DAB Detection System (Diagnostic Biosystems) was used for primary antibody visualization.

The method employed in this study has been described in our previous work. ${ }^{10,11}$ Briefly, sections of tissue ( 4 to $5 \mu \mathrm{m}$ thick) were deparaffinized and washed with distilled water. Antigen retrieval was performed using a microwave. The sections were then

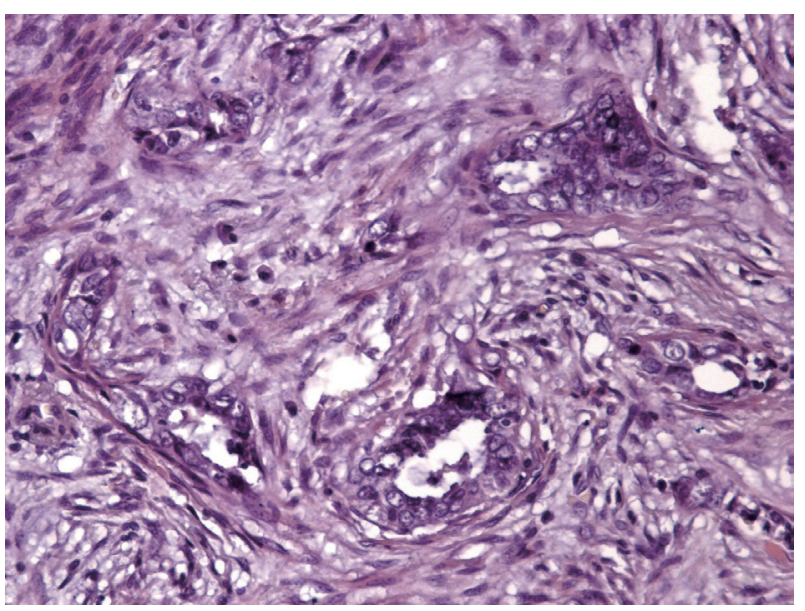

Fig. 1. Specific microcystic, elongated and fragmented pattern changes: endometrioid carcinoma showing eosinophilic glands with microcystic transformation embedded in fibromyxoid stroma and cancer cell complexes with some areas resembling vascular invasion.

Table 1. Clinicopathological characteristics of the patients

\begin{tabular}{lccr}
\hline Characteristic & $\begin{array}{c}\text { MELF-positive } \\
\text { group }(n=49)\end{array}$ & $\begin{array}{c}\text { MELF-negative } \\
\text { group }(n=51)\end{array}$ & p-value \\
\hline Age (yr) & $64.2 \pm 4.8$ & $63.8 \pm 5.2$ & $.982^{\mathrm{a}}$ \\
FIGO stage & & & $.942^{\mathrm{b}}$ \\
$\quad$ I & 12 & 11 & \\
II & 23 & 25 & \\
$\quad$ III & 14 & 15 & $.855^{\mathrm{b}}$ \\
Tumor grade & & & \\
$\quad$ G1 & 20 & 22 & \\
G2 & 23 & 22 & \\
$\quad$ G3 & 6 & 7 & \\
Lymphovascular invasion & & & \\
$\quad$ Present & 29 & 22 & \\
$\quad$ Absent & 20 & 29 & \\
Myometrial invasion (\%) & & & \\
$\quad<50$ & 10 & $99^{c}$ \\
$\quad>50$ & 39 & 42 & \\
\hline
\end{tabular}

MELF, microcystic, elongated and fragmented; FIGO, International Federation of Gynecology and Obstetrics.

aMann-Whitney U test; 'bruskal-Wallis test; 'Fisher's two-tailed exact test. 
allowed to cool and endogenous peroxidase blocking was performed in $5 \%$ hydrogen peroxide. Blocking of nonspecific antibody binding was carried out in $5 \%$ casein. Sections were washed and incubated in a moist chamber at room temperature with corresponding primary antibodies followed by incubation with anti-mouse horseradish peroxidase secondary antibodies. The reaction product was visualized after 3,3-diaminobenzidine (DAB) staining for 5 minutes followed by Mayer's hematoxylin counterstaining. ${ }^{10,11}$

\section{Morphometry}

The morphometrical analysis was carried out using package NIS-Elements. The tumor invasion zone was photographed using a microscope (Nikon Eclipse 50i, Nikon, Tokyo, Japan) with a digital camera (DS-Fi2) in 5 non-overlapping high-power fields (HPF; $\times 400$ magnification) of maximum expression for each marker. Expression of VEGF and galectin-1 in cell counting was analyzed using the function "measure." The number of positive cells was expressed as a percentage of the total number of cells in the epithelial component of the tumor.

MVD was presented as the area and number of vessels per $\mathrm{mm}^{2}$. The number of vessels was counted in 5 non-overlapping HPF in areas of "hot spots" and inverted into $\mathrm{mm}^{2}$ using the formula:

$$
\mathrm{N}=\overline{\mathrm{X}} n \times 1000000 / 118947.07
$$

where $\mathrm{N}=$ number of vessels per $\mathrm{mm}^{2} ;(\overline{\mathrm{X}}) n=$ mean number of vessels; and 118947,07 = area of one $\operatorname{HPF}\left(\mu \mathrm{m}^{2}\right)$.

The area of the vessels was counted in 5 non-overlapping HPFs in regions of "hot spots" using the function "area." Area of vessels after the count was inverted into $\mathrm{mm}^{2}$ using the formula:

$S=\bar{X}_{s} \times 1000000 / 118947.07$

where $\mathrm{S}=$ area of vessels per $1 \mathrm{~mm}^{2} ; \overline{\mathrm{X}}_{s}=$ mean area of vessels; and $118947,07=$ area of one $\operatorname{HPF}\left(\mu \mathrm{m}^{2}\right)$.

\section{Statistical analysis}

Power analysis (power $=80 \%, \alpha=0.05$ ) during the pilot study, in which parameters were received from four cases with MELF pattern and four without the pattern in $5 \mathrm{HPF}$, revealed that the minimum number of subjects should be 18 . The data were presented as the median, lower and upper quartiles. The MannWhitney test was used for comparing the study groups based on the evaluated criteria. The Spearman correlation test with Chaddock scale was used to perform correlation analysis. The hazard ratio (HR) with a $95 \%$ confidence interval $(95 \% \mathrm{CI})$ was determined for overall survival (OS) and disease-free survival (DFS) using univariate and multivariate Cox proportional hazards regres- sion models. A p $<0.05$ was considered statistically significant. GraphPad Prism v.7.29 (GraphPad Software Inc., San Diego, CA, USA) and R Software v 3.4.0 were used for analysis (R Foundation for Statistical Computing, Vienna, Austria).

\section{Ethics statement}

Immunohistochemical study was undertaken at the Gomel State Medical University, Belarus, with informed consent from all patients and with ethical approval from the Institutional Review Board (Gomel State Medical University), Gomel, Belarus.

\section{RESULTS}

\section{Galectin-1 expression in EA tissue}

Galectin-1 was observed to be diffusely expressed by epithelial cancer cells, stromal cancer cells and vessels in both groups. However, the intensity of the expression of this marker in the MELF negative group was significantly lower $(\mathrm{p}<.001)$ than the MELF-positive group (Fig. 2A, B). The median galectin-1 expression in the MELF pattern-positive and -negative groups were $78.6 \%(40.1 \%-88.3 \%)$ and $34.2 \%(24.4 \%-55.5 \%)$, respectively. The Mann-Whitney test showed statistically significant differences in galectin-1 expression in both groups $(\mathrm{p}<.001)$.

\section{VEGF}

The expression of VEGF was also observed across all cancer cells and stromal elements. In the MELF-negative group, predominantly moderate expression of this marker was observed (Fig. 2C). In the MELF pattern positive group, the expression was substantially intense (Fig. 2D). Median VEGF expression in the MELF-positive group was $81.4 \%(58.1 \%-86.3 \%)$ and in the group without MELF pattern was $53.0 \%$ (47.6\%-75.4\%). The VEGF expression difference between the two groups was statistically significant $(\mathrm{p}<.001)$.

\section{Microvessel density}

In various regions of the MELF pattern, a low number of vessels was observed. In the group without MELF pattern, vessels were situated in small groups, and lymphovascular invasion of EA was mostly absent (Fig. 2E). A significant number of microvessels was seen in the MELF pattern cases in "hot spots" regions with unusual lumens. This was often observed in areas of lymphovascular invasion (Fig. 2F).

We also measured the number of vessels per $\mathrm{mm}^{2}$ in these tissue sections. We found that the median values in MELF-negative and MELF-positive groups were 101.6 vessels $/ \mathrm{mm}^{2}$ (range, 

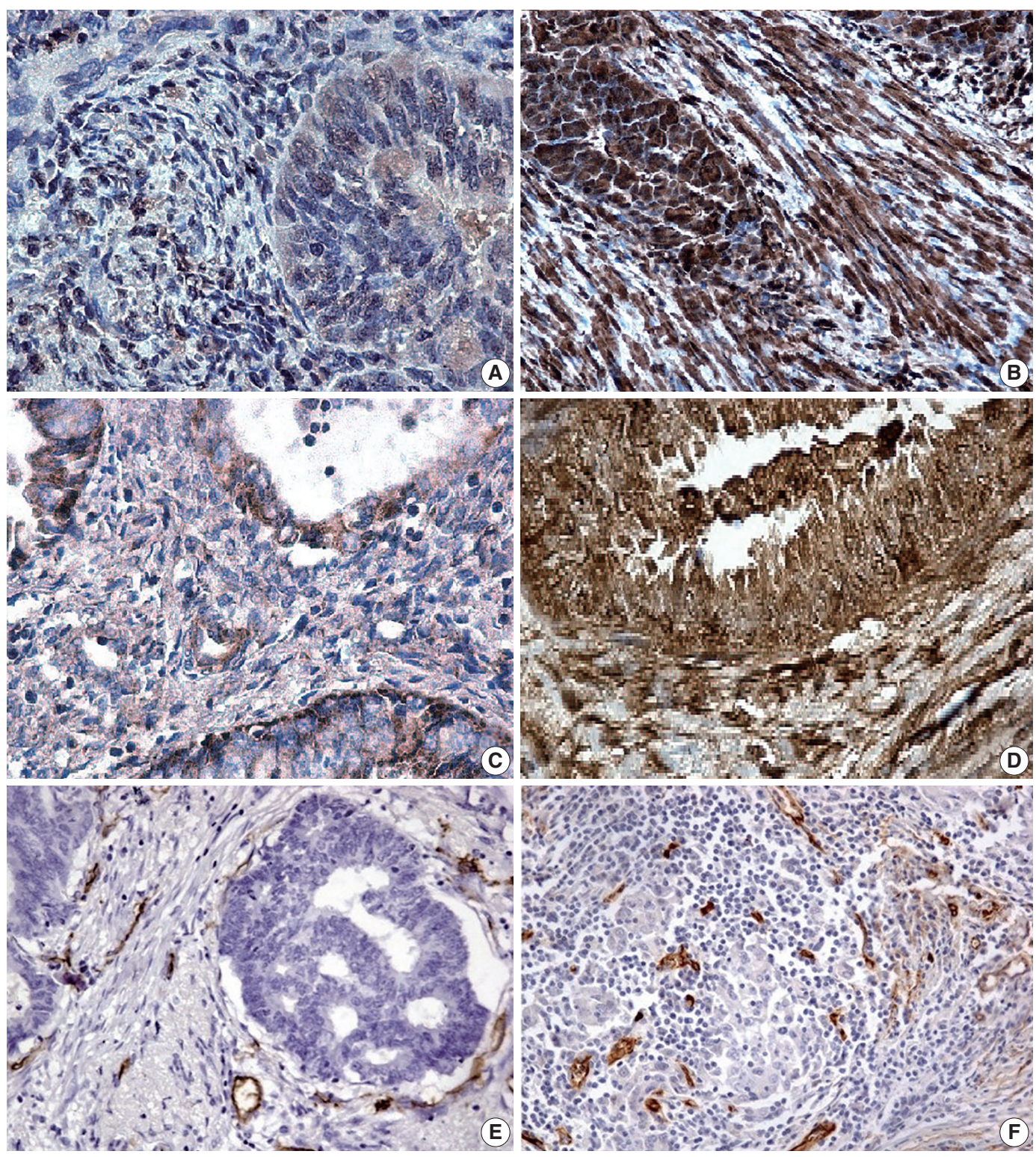

Fig. 2. (A) A few positive glandular cells (microcystic, elongated and fragmented [MELF]-negative group). (B) All glandular cells positive for galectin-1 expression (MELF-positive group) in endometrioid carcinoma. (C) Positive local areas of cytoplasmic expression of vascular endothelial growth factor (VEGF; MELF-negative group). (D) Intense glandular and cytoplasmic (MELF-positive group) expression of VEGF in glands of the endometrioid carcinoma. Low (normal stroma) $(E)$ and increased number of vessels (F) in fibromyxoid stroma situated around cancer cells forming gland without lumens.

90.1 to 127.9 vessels $/ \mathrm{mm}^{2}$ ) and 134.5 vessels $/ \mathrm{mm}^{2}$ (range, 98.5 to 156.7 vessels $/ \mathrm{mm}^{2}$ ), respectively, where the latter group showed significantly higher presence than the former $(\mathrm{p}<.010)$.

The area of vessels per $\mathrm{mm}^{2}$ was also significantly higher $(\mathrm{p}<$ 0.001 ) in the stomal changes of the MELF-positive group $\left(4,788.3 \mu \mathrm{m}^{2} / \mathrm{mm}^{2}\right.$; range, $3,087.3$ to $\left.5,130.4 \mu \mathrm{m}^{2} / \mathrm{mm}^{2}\right)$ compared to the group without such specific stromal changes (3,037.3 $\mathrm{mm}^{2} / \mathrm{mm}^{2}$; range, $2,508.3$ to $\left.5,130.4 \mu \mathrm{m}^{2} / \mathrm{mm}^{2}\right)$.

\section{Correlation analyses}

We also analyzed the associations among expressions of the MELF pattern, galectin-1, VEGF, area and number of vessels per $\mathrm{mm}^{2}$, the outcome of EA (survival or death), and the number of days of survival (Fig. 3). For instance, galectin-1 demonstrated a moderate positive correlation with the area of vessels per $\mathrm{mm}^{2}(\mathrm{p}<.001, \mathrm{r}=0.69)$ and VEGF expression $(\mathrm{p}<.001, \mathrm{r}=$ $0.67)$. Furthermore, galectin-1 expression had a highly positive correlation with the outcome of $\mathrm{EA}(\mathrm{p}<.001, \mathrm{r}=0.78)$ and a high- 
ly negative one with the number of days of survival $(\mathrm{p}<.001, \mathrm{r}=$ $-0.84)$.

The expression of VEGF had a highly positive correlation with the outcome of EA $(p<.001, r=0.85)$ and a highly negative correlation with the number of days of survival $(\mathrm{p}<.001, \mathrm{r}=-0.78)$. The number of vessels per $\mathrm{mm}^{2}$ revealed moderately positive correlations with galectin- $1(\mathrm{p}<.001, \mathrm{r}=0.54)$, area of vessels per $\mathrm{mm}^{2}(\mathrm{p}<.001, \mathrm{r}=0.61)$, and VEGF $(\mathrm{p}<.001, \mathrm{r}=0.56)$; a highly

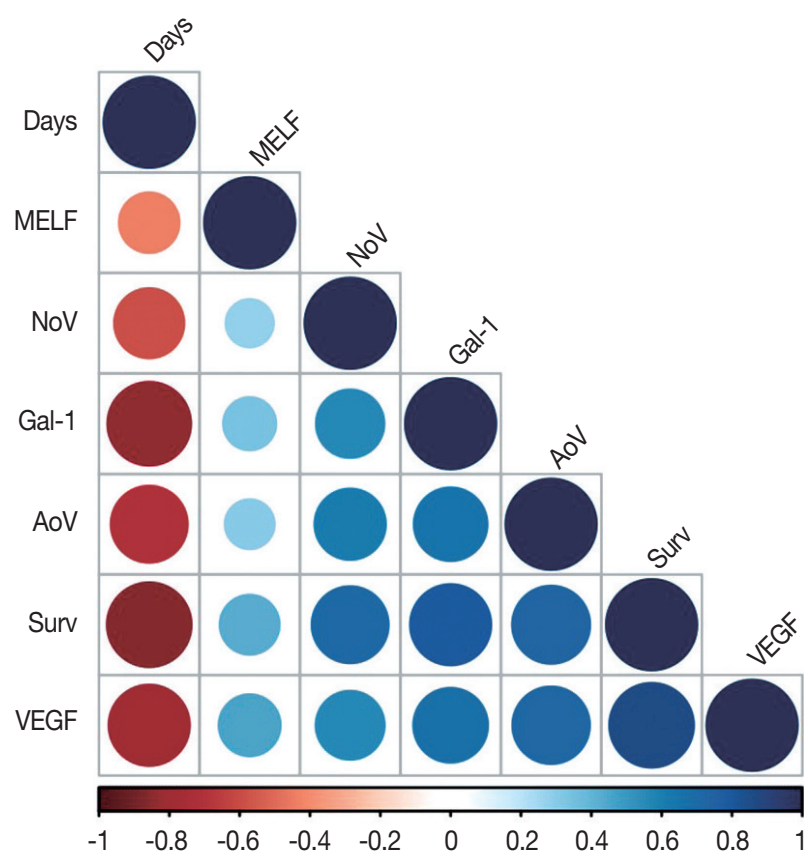

Fig. 3. A correlation plot. Days, number of days of survival from surgical treatment to death or end of observation; MELF, microcystic, elongated and fragmented pattern; NoV, number of vessels per mm²; Gal-1, galectin-1 expression; AoV, area of vessels per mm²; Surv, survival (survival or death); VEGF, vascular endothelial growth factor expression. positive correlation with the outcome of EA ( $<<.001, r=0.71)$; and a moderately negative correlation with the number of days of survival $(\mathrm{p}<.001, \mathrm{r}=-0.59)$.

A highly positive correlation was observed between the area of vessels per $\mathrm{mm}^{2}$ and the outcome of EA $(\mathrm{p}<.001, \mathrm{r}=0.73)$ and VEGF expression $(\mathrm{p}<.001, \mathrm{r}=0.72)$. A highly negative correlation was found between the area of vessels per $\mathrm{mm}^{2}$ and the number of days of survival $(\mathrm{p}<.001, \mathrm{r}=-0.71)$.

MELF pattern had a slightly negative correlation with the number of days of survival $(\mathrm{p}<.001, \mathrm{r}=-0.47)$ and slightly positive correlation with galectin-1 expression $(p<.001, r=0.39)$, the area of vessels per $\mathrm{mm}^{2}(\mathrm{p}<.001, \mathrm{r}=0.36)$, the outcome of EA $(\mathrm{p}<.001, \mathrm{r}=0.42)$, and VEGF expression $(\mathrm{p}<.001, \mathrm{r}=0.39)$.

\section{Survival analysis}

An OS univariate survival analysis was performed, indicating that all parameters except age play a role in the survival of patients with EA (Table 2). However, a multivariate Cox proportional hazard regression analysis of OS revealed that only the area of vessels per $\mathrm{mm}^{2}$ (HR, 1.018; 95\% CI, 1.002 to 1.033), galectin-1 (HR, 1.049; 95\% CI, 1.025 to 1.074$)$ and VEGF (HR, 1.049; 95\% CI, 1.022 to 1.077) play key roles in survival irrespective of MELF pattern (Table 2).

A univariate analysis was performed, revealing that all parameters except age and tumor grade play a role in DFS of patients with EA (Table 3). However, our multivariate Cox proportional hazard regression analysis demonstrated that only MELF pattern (HR, 1.018; 95\% CI, 1.002 to 1.033) and galectin-1 expression (HR, 1.049; 95\% CI, 1.025 to 1.074$)$ were significant predictors of DFS (Table 3).

Table 2. The univariate and multivariate Cox regression analysis results for overall survival

\begin{tabular}{|c|c|c|c|c|c|c|}
\hline \multirow{2}{*}{ Parameter } & \multicolumn{3}{|c|}{ Univariable analysis } & \multicolumn{3}{|c|}{ Multivariable analysis } \\
\hline & Hazard ratio & $95 \% \mathrm{Cl}$ & $p$-value & Hazard ratio & $95 \% \mathrm{Cl}$ & $p$-value \\
\hline Age & 1.012 & $0.983-1.041$ & .435 & 0.992 & $0.959-1.027$ & .668 \\
\hline FIGO & 1.993 & $1.361-2.918$ & $<.001$ & 1.304 & $0.774-2.292$ & .332 \\
\hline Grade & 2.201 & $1.469-3.297$ & $<.001$ & 1.332 & $0.776-2.251$ & .304 \\
\hline Lymphovascular invasion & 1.084 & $1.052-1.118$ & $<.001$ & 1.010 & $0.942-1.049$ & .624 \\
\hline Myometrial invasion & 1.032 & $1.011-1.048$ & $<.001$ & 1.028 & $0.911-1.037$ & .698 \\
\hline MELF pattern & 4.115 & $2.240-7.558$ & $<.001$ & 1.748 & $0.870-3.513$ & .117 \\
\hline No. of vessels & 1.036 & $1.026-1.045$ & $<.001$ & 1.000 & $0.999-1.000$ & .623 \\
\hline Area of vessels & 1.001 & $1.001-1,001$ & $<.001$ & 1.018 & $1.002-1.033$ & .025 \\
\hline VEGF & 1.078 & $1.058-1.098$ & $<.001$ & 1.049 & $1.022-1.077$ & $<.001$ \\
\hline Galectin-1 & 1.071 & $1.054-1089$ & $<.001$ & 1.049 & $1.025-1.074$ & $<.001$ \\
\hline
\end{tabular}

$\mathrm{Cl}$, confidence interval; FIGO, International Federation of Gynecology and Obstetrics; MELF, microcystic, elongated and fragmented; VEGF, vascular endothelial growth factor. 
Table 3. The univariate and multivariate Cox regression analysis results for disease-free survival

\begin{tabular}{|c|c|c|c|c|c|c|}
\hline \multirow{2}{*}{ Parameter } & \multicolumn{3}{|c|}{ Univariable analysis } & \multicolumn{3}{|c|}{ Multivariable analysis } \\
\hline & Hazard ratio & $95 \% \mathrm{Cl}$ & $p$-value & Hazard ratio & $95 \% \mathrm{Cl}$ & $p$-value \\
\hline Age & 1.013 & $0.977-1.049$ & .495 & 0.984 & $0.930-1.042$ & .584 \\
\hline FIGO & 2.330 & $1.456-3.728$ & $<.001$ & 0.960 & $0.473-1.948$ & .910 \\
\hline Grade & 1.512 & $0.928-2.463$ & .097 & 0.886 & $0.409-1.846$ & .714 \\
\hline Lymphovascular invasion & 1.083 & $1.043-1.124$ & $<.001$ & 0.986 & $0.940-1.032$ & .544 \\
\hline Myometrial invasion & 1.014 & $1.012-1.052$ & $<.001$ & 0.978 & $0.937-1.048$ & .587 \\
\hline MELF pattern & 4.510 & $1.938-6.342$ & $<.001$ & 3.472 & $3.109-3.877$ & $<.001$ \\
\hline No. of vessels & 1.026 & $1.014-1.038$ & $<.001$ & 1.007 & $0.989-1.025$ & .441 \\
\hline Area of vessels & 1.002 & $1.001-1.004$ & $<.001$ & 1.000 & $0.999-1.000$ & .431 \\
\hline VEGF & 1.049 & $1.029-1.068$ & $<.001$ & 1.004 & $0.968-1.043$ & .813 \\
\hline Galectin-1 & 1.037 & $1.023-1.057$ & $<.001$ & 1.050 & $1.016-1.084$ & .003 \\
\hline
\end{tabular}

$\mathrm{Cl}$, confidence interval; FIGO, International Federation of Gynecology and Obstetrics; MELF, microcystic, elongated and fragmented; VEGF, vascular endothelial growth factor.

\section{DISCUSSION}

In this study, we investigated the associations between galectin-1, VEGF, MVD, area of vessels, and survival outcome of EA patients with and without a MELF pattern. We identified 49 patients out of 100 with a MELF pattern and 51 patients without this stromal change who were observed throughout 36 months from admission to the hospital. In this immunohistochemical study, we demonstrated differential expressions of galectin-1, VEGF and measurement of MVD in cases with and without MELF pattern.

In the last two decades, several galectin family members have emerged as versatile modulators of tumor progression. Galectin-1 expression is also frequently reported to be increased in the reproductive system as well as the placenta. There is ample evidence that malignant transformation is accompanied by elevated galectin-1 levels. ${ }^{12}$ Jeschke et al. ${ }^{13}$ reported a statistically significant increase of galectin-1 expression in EA with grade 3 and stages III/VI (FIGO) compared to grades 1 and 2 and FIGO I/II. However, no data are available on the expression of galectin-1 in EA with different types of stromal changes and survival. In our study, we found that galectin-1 expression is higher in EA with a MELF pattern compared to cases without a MELF pattern. Our observation of positive correlations between galectin- 1 expression and MELF pattern (fibromyxoid changes) and EA outcome agrees with our previous report and with a report by Sandberg et al., ${ }^{14}$ in which a correlation was shown between an increased galectin-1 expression and fibrotic reaction in tumor progression in the stroma in colon cancer. This was accompanied by decreased immune cell infiltration, indicating a pro-cancerous role of galectin-1..$^{14,15}$

VEGF was originally identified as a multifunctional cytokine in angiogenesis and lymphangiogenesis. ${ }^{16}$ In cancer angiogenesis, VEGF promotes the mobilization of inflammatory cells to the tumor site, maintaining the local inflammatory process and inducing the synthesis of proangiogenic factors by endothelial cells, platelets, smooth muscle cells, inflammatory cells, fibroblasts, and tumor cells. ${ }^{17}$ This interaction between tumor and stromal cells may result in an increased VEGF expression with cancerassociated fibroblasts being the primary source of VEGF. ${ }^{18} \mathrm{Im}$ mature cells of the tumor microenvironment have higher secretion of VEGF than mature cells. ${ }^{19}$ We suggest that this is the reason that in our study, MELF pattern stroma had higher expression of VEGF than normal stroma in EA.

Angiogenesis is also known to play crucial roles in the malignant behavior of tumors by increasing oxygen and nutrient supply to cancer cells where the neo-vasculatures form an irregular network of capillaries. In addition, such abnormal vasculatures are important as a pathway for cancer cell metastasis. ${ }^{20}$ Accumulated evidence indicates that tumor angiogenesis assessed by blood MVD is associated with advanced clinicopathological parameters and poor prognostic outcomes in different types of cancers. ${ }^{21}$ MVD analysis demonstrates high prognostic value in cancers of different locations, such as renal, cervical, colorectal and others. ${ }^{22,23}$ In MELF pattern positive EA, an increased number and area of vessels were observed, which agrees with the previously reported study by Joehlin-Price et al. ${ }^{24}$ on the high frequency of lymph node metastasis.

A moderate correlation between the area of vessels per $\mathrm{mm}^{2}$ and galectin-1 may be due to the cross-talk between galectinglycan interactions and vascular compartments. ${ }^{25}$ The correlation between galectin-1 and VEGF may be associated with the mirroring effects of these proangiogenic factors. ${ }^{26}$ Galectin-1 expression in EA may be a prognostic factor in OS of patients, an 
observation that was reported by Wu et al. ${ }^{27}$ That study reported predictive values of this parameter for cholangiocarcinoma, hepatocellular carcinoma, gingival squamous cell carcinoma, head and neck squamous cell carcinoma, renal cell carcinoma, nonsmall cell lung cancer, gastric carcinoma and glioblastoma multiforme. $^{27}$

VEGF as the main proangiogenic factor and immune suppressive factor of the tumor microenvironment correlates with the survival of patients suffering from EA. Goel and Mercurio ${ }^{28}$ reported a strong expression of VEGF in endothelial cells in the stromal microvessels adjacent to malignant glands, which has been reported to be significantly associated with tumor progression and metastasis.

A positive correlation between MVD and galectin-1/VEGF expression suggested that these parameters play a role in angiogenesis. Additionally, a highly positive correlation between galectin-1 expression and poor survival, as well as MVD and poor survival, suggested that these factors may have a considerable predictive role in survival. ${ }^{29}$

The presence of a MELF pattern revealed low correlations with the above parameters of proangiogenic response in EA. However, a low negative correlation of MELF pattern with the number of days of survival and a moderate positive correlation with the outcome of the disease may show that MELF pattern may be an independent prognostic criterion of patients' OS.

The results of multivariate survival analyses showed that MELF pattern plays a role in DFS of patients with EA, but not in OS. However, our univariate analysis demonstrated a significant effect of MELF pattern on both OS and DFS. Previous reports have been contradictory on the role of MELF pattern. Sanci et al. ${ }^{6}$ and our previous work reported a positive association, but other authors showed that the presence of a MELF pattern plays no role in OS. Our study also demonstrated MELF pattern as a predictive factor of DFS that may be associated with more frequent lymph node involvement in cases of MELF pattern presence. The absence of influence of FIGO and tumor grade on DFS and OS in our study could be associated with homogeneity of the comparison groups for these parameters. However, our inclusion and exclusion criteria eliminated any potential bias. The systematic review of Prodromidou et al. ${ }^{30}$ revealed that most of the published literature describing the role of MELF patterns in patients' survival did not study large cohorts of patients, which may be a factor in the inconclusive observations.

Potential limitations of this study are its case-control nature with a relatively small volume of cases due to the rare incidence of MELF patterns in EA. Despite these limitations, a number of study population characteristics mitigated the weaknesses and increased the validity of our results. One characteristic was the availability of excellent follow-up data. Another was that histopathological analyses made by experienced pathologists. Others included that uniform surgical and radiological treatment was provided by the same surgical teams in two oncological dispensaries and followed protocols of diagnostics and treatment of oncological diseases of the Republic of Belarus.

In conclusion, this study presented an increase of MVD in areas of "hot spots" and angiogenic factors such as VEGF and galectin-1 in cases of EA with MELF presence. Hence, these biomarkers may be used as potential targets for therapy of EA, especially in the implementation of personalized medicine in cases with such specific stromal changes. Our univariate survival analysis revealed MELF pattern presence to be an independent predictor in overall EA survival, but the multivariate analysis did not show that its combination with previously described parameters plays a role in survival. Thus, a large case-control study should be conducted to confirm our findings.

$$
\begin{aligned}
& \text { ORCID } \\
& \text { Dmitry Aleksandrovich Zinovkin: } \\
& \text { https://orcid.org/0000-0002-3808-8832 } \\
& \text { Sergey Leonidovich Achinovich: } \\
& \text { https://orcid.org/0000-0002-0172-0320 } \\
& \text { Mikhail Grigoryevich Zubritskiy: } \\
& \text { https://orcid.org/0000-0001-5355-9076 } \\
& \text { Jacqueline Linda Whatmore: } \\
& \text { https://orcid.org/0000-0002-8753-1116 } \\
& \text { Md Zahidul Islam Pranjol: } \\
& \text { https://orcid.org/0000-0002-6164-6281 }
\end{aligned}
$$

\section{Author Contributions}

Conceptualization: DAZ, MZIP.

Data curation: SLA, MGZ.

Formal analysis: SLA, MGZ.

Funding acquisition: JLW (partial).

Investigation: DAZ, MZIP.

Methodology: DAZ.

Project administration: DAZ, MZIP.

Resources: SLA, MGZ.

Software: DAZ.

Supervision: JLW, MZIP.

Validation: SLA, MGZ.

Visualization: SLA, MGZ. 
Writing—original draft: DAZ, MZIP.

Writing—review \& editing: DAZ, JLW, MZIP.

\section{Conflicts of Interest}

The authors declare that they have no potential conflicts of interest.

\section{Funding}

This research was partially supported by FORCE Cancer Charity, Devon, UK and initial grant of the Gomel State Medical University "Development and implementation of a prognostic model of the endometrioid adenocarcinoma of the uterine body based on pathological parameters of the tumor microenvironment," registration number 20190038, registration date 24.01.2019, Gomel, Belarus.

\section{REFERENCES}

1. Horn LC, Meinel A, Handzel R, Einenkel J. Histopathology of endometrial hyperplasia and endometrial carcinoma: an update. Ann Diagn Pathol 2007; 11: 297-311.

2. Banas T, Pitynski K, Okon K, Winiarska A. Non-endometrioid and high-grade endometrioid endometrial cancers show DNA fragmentation factor 40 (DFF40) and B-cell lymphoma 2 protein (BCL2) underexpression, which predicts disease-free and overall survival, but not DNA fragmentation factor 45 (DFF45) underexpression. BMC Cancer 2018; 18: 418.

3. Reeves KW, Carter GC, Rodabough RJ, et al. Obesity in relation to endometrial cancer risk and disease characteristics in the Women's Health Initiative. Gynecol Oncol 2011; 121: 376-82.

4. SGO Clinical Practice Endometrial Cancer Working Group, Burke WM, Orr J, et al. Endometrial cancer: a review and current management strategies: part II. Gynecol Oncol 2014; 134: 393-402.

5. Murray SK, Young RH, Scully RE. Unusual epithelial and stromal changes in myoinvasive endometrioid adenocarcinoma: a study of their frequency, associated diagnostic problems, and prognostic significance. Int J Gynecol Pathol 2003; 22: 324-33.

6. Sanci M, Güngördük K, Gülseren V, et al. MELF pattern for predicting lymph node involvement and survival in grade I-II endometrioid-type endometrial cancer. Int J Gynecol Pathol 2018; 37: 17-21.

7. Kihara A, Yoshida H, Watanabe R, et al. Clinicopathologic association and prognostic value of microcystic, elongated, and fragmented (MELF) pattern in endometrial endometrioid carcinoma. Am J Surg Pathol 2017; 41: 896-905.

8. Pelletier MP, Trinh VQ, Stephenson P, et al. Microcystic, elongated, and fragmented pattern invasion is mainly associated with isolated tumor cell pattern metastases in International Federation of Gynecology and Obstetrics grade I endometrioid endometrial cancer. Hum Pathol 2017; 62: 33-9.

9. Stewart CJ, Brennan BA, Leung YC, Little L. MELF pattern invasion in endometrial carcinoma: association with low grade, myoinvasive endometrioid tumours, focal mucinous differentiation and vascular invasion. Pathology 2009; 41: 454-9.

10. Zinovkin DA, Pranjol MZI, Petrenyov DR, Nadyrov EA, Savchenko OG. The potential roles of MELF-pattern, microvessel density, and VEGF expression in survival of patients with endometrioid endometrial carcinoma: a morphometrical and immunohistochemical analysis of 100 cases. J Pathol Transl Med 2017; 51: 456-62.

11. Zinovkin D, Pranjol MZ. Tumor-infiltrated lymphocytes, macrophages, and dendritic cells in endometrioid adenocarcinoma of corpus uteri as potential prognostic factors: an immunohistochemical study. Int J Gynecol Cancer 2016; 26: 1207-12.

12. Zhu X, Wang K, Zhang K, et al. Galectin-1 knockdown in carcinomaassociated fibroblasts inhibits migration and invasion of human MDA-MB-231 breast cancer cells by modulating MMP-9 expression. Acta Biochim Biophys Sin (Shanghai) 2016; 48: 462-7.

13. Jeschke U, Walzel H, Mylonas I, et al. The human endometrium expresses the glycoprotein mucin- 1 and shows positive correlation for Thomsen-Friedenreich epitope expression and galectin-1 binding. J Histochem Cytochem 2009; 57: 871-81.

14. Sandberg TP, Oosting J, van Pelt GW, Mesker WE, Tollenaar R, Morreau $\mathrm{H}$. Molecular profiling of colorectal tumors stratified by the histological tumor-stroma ratio: increased expression of galectin-1 in tumors with high stromal content. Oncotarget 2018; 9: 31502-15.

15. Zinovkin DA, Pranjol MZ, Bilsky IA, Zmushko VA. Tumor-associated T-lymphocytes and macrophages are decreased in endometrioid endometrial carcinoma with MELF-pattern stromal changes. Cancer Microenviron 2018; 11: 107-14.

16. Albini A, Bruno A, Noonan DM, Mortara L. Contribution to tumor angiogenesis from innate immune cells within the tumor microenvironment: implications for immunotherapy. Front Immunol 2018; 9: 527.

17. Melincovici CS, Boşca AB, Şuşman S, et al. Vascular endothelial growth factor (VEGF): key factor in normal and pathological angiogenesis. Rom J Morphol Embryol 2018; 59: 455-67.

18. Tao L, Huang G, Song H, Chen Y, Chen L. Cancer associated fibroblasts: an essential role in the tumor microenvironment. Oncol Lett 2017; 14: 2611-20.

19. Li YL, Zhao H, Ren XB. Relationship of VEGF/VEGFR with immune and cancer cells: staggering or forward? Cancer Biol Med 2016; 13: 206-14.

20. Miyata Y, Sakai H. Reconsideration of the clinical and histopatho- 
logical significance of angiogenesis in prostate cancer: Usefulness and limitations of microvessel density measurement. Int J Urol 2015; 22: 806-15.

21. Jilaveanu LB, Puligandla M, Weiss SA, et al. Tumor microvessel density as a prognostic marker in high-risk renal cell carcinoma patients treated on ECOG-ACRIN E2805. Clin Cancer Res 2018; 24: 217-23.

22. Hu X, Liu H, Ye M, Zhu X. Prognostic value of microvessel density in cervical cancer. Cancer Cell Int 2018; 18: 152.

23. Mezheyeuski A, Nerovnya A, Bich T, Tur G, Ostman A, Portyanko A. Inter- and intra-tumoral relationships between vasculature characteristics, GLUT1 and budding in colorectal carcinoma. Histol Histopathol 2015; 30: 1203-11.

24. Joehlin-Price AS, McHugh KE, Stephens JA, et al. The microcystic, elongated, and fragmented (MELF) pattern of invasion: a single institution report of 464 consecutive FIGO grade 1 endometrial endometrioid adenocarcinomas. Am J Surg Pathol 2017; 41: 49-55.

25. Kamili NA, Arthur CM, Gerner-Smidt C, et al. Key regulators of galectin-glycan interactions. Proteomics 2016; 16: 3111-25.

26. Chen J, Tang D, Wang S, et al. High expressions of galectin-1 and VEGF are associated with poor prognosis in gastric cancer patients. Tumour Biol 2014; 35: 2513-9.

27. Wu R, Wu T, Wang K, et al. Prognostic significance of galectin-1 expression in patients with cancer: a meta-analysis. Cancer Cell Int 2018; 18: 108.

28. Goel HL, Mercurio AM. VEGF targets the tumour cell. Nat Rev Cancer 2013; 13: 871-82.

29. Wang JZ, Xiong YJ, Man GC, Chen XY, Kwong J, Wang CC. Clinicopathological and prognostic significance of blood microvessel density in endometrial cancer: a meta-analysis and subgroup analysis. Arch Gynecol Obstet 2018; 297: 731-40.

30. Prodromidou A, Vorgias G, Bakogiannis K, Kalinoglou N, Iavazzo C. MELF pattern of myometrial invasion and role in possible endometrial cancer diagnostic pathway: a systematic review of the literature. Eur J Obstet Gynecol Reprod Biol 2018; 230: 147-52. 